\title{
Why is the Shaky Bridge so Shaky?
}

\section{Deirdre O’Donnell}

\author{
Civil and Environmental Engineering, School of Engineering, UCC
}

"The Shaky Bridge. Officially the shakiest bridge in Ireland".

This is the claim of a poster ad campaign for Murphy's Stout, and it demonstrates the legendary status attached to Cork's most famous and most loved dynamic structure.

\section{History of Structure}

There are only three suspension bridges in Ireland; at Birr and Kinnitty Castles, both in Co. Offaly, and Cork's own Daly's Bridge. The $87 \mathrm{~m}$ meter long steel suspension bridge spans the River Lee, shown in Figure 1, connecting the Mardyke and Sunday's Well areas just east of Cork city centre. Constructed in 1926 to replace a ferry crossing at the location, it was manufactured by David Rowell \& Co. Ltd. of London to the specifications of then Cork City engineer Stephen William Farrington. Named Daly's Bridge for James Daly, a wealthy benefactor of the project, today it is better known locally as 'The Shaky Bridge' for its perceived 'shakiness'.

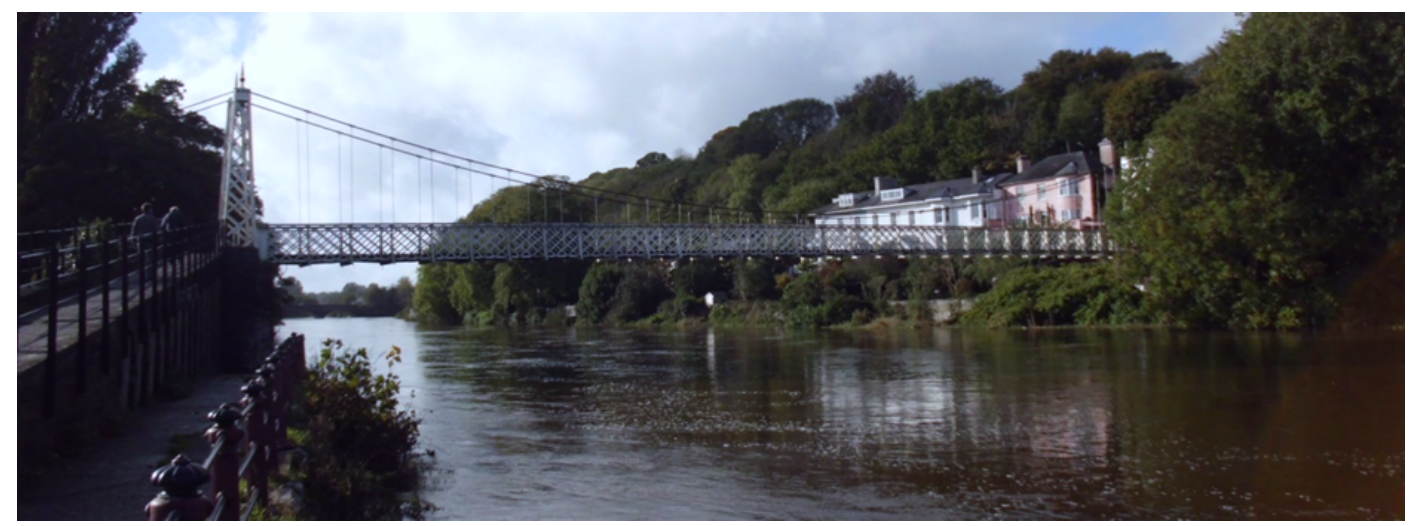

Figure 1: Daly's Bridge, River Lee, Cork. Source: Author

Despite being renowned for its dynamic nature, very little work has been done to investigate the reason for or the degree of lively behaviour for this historic steel structure. According to local knowledge, some refurbishment work was undertaken in the 1980s, which involved replacement of the timber walkway and which residents say reduced the level of movement in the bridge. Some improvements were also made to the steel parapets and towers, and this can be seen by the varying degrees of degradation in an otherwise homogenous material. 


\section{Structural Health Monitoring}

"The process of implementing a damage identification strategy for aerospace, civil and mechanical engineering infrastructure is referred to as structural health monitoring (SHM)."-'The fundamental axioms of SHM', Worden et al (2007)

The monitoring of structures in order to assess their capacity to fulfil their intended purpose has been taking place to some extent as long as structures have existed, but the formalisation of this field into a branch of engineering in its own right has truly come to prominence in recent years. It has become so well established that there now exist 'axioms' of SHM; fundamental truths on which a process can be implemented. It can also be summarised as a four step process:

1. The identification of damage existence

2. The determination of damage location

3. The identification of the damage type

4. The determination of the damage severity

Structural Health Monitoring (SHM) involves the monitoring of bridges and other structures over time, with the aim of identifying the presence, location and extent of damage with a final view to predicting the lifespan of the structure. SHM focuses on developing technologies and systems that assess integrity of structures such as buildings, bridges, aero-space structures and off-shore oil rigs. SHM systems can be employed in design validation, detection of damage or deterioration, safety assessment, instruction on prioritisation of repair and maintenance, evaluating repair works or simply to add to the knowledge base of the broader engineering field. So while this project focuses on Daly's Bridge, it also aims to outline a method that can be used to assess similar structures, while contributing to the knowledge base for this growing field of engineering.

\section{Perceptibility of Motion to Pedestrians}

Many footbridges are slender structures with natural frequencies (that is, the frequency at which the structure will vibrate without external forces) that often lie in the domain of the dominant frequencies of walking pedestrians. Naturally the most frequent and often dominant dynamic load for a footbridge is pedestrians walking. It is believed that excessive vibrations in footbridges are caused by near resonance of one or more modes of vibration due to the similarity of the pedestrians' footsteps and the bridge's natural frequency.

Pedestrian forcing can lead to vibrations, both laterally and vertically, that are perceptible and sometimes uncomfortable to humans. It is an issue of serviceability that has come under much interest recently, due to high profile cases such as the Millennium Bridge in London. This bridge had to be closed to the public shortly after its official opening, 
as the crowds of pedestrians had an unanticipated effect on the bridge, causing extreme motions which many felt was unsafe. There exist solutions for when vibrations fall outside of the range of acceptability; in the case of the Millennium Bridge, it was retrofitted with tuned mass dampers and fluid viscous dampers which, respectively dealt with vertical and horizontal movement by absorbing the vibrations. The lack of design codes, the standards engineers must adhere to when designing a structure, to address this issue of serviceability led to this mishap and this problem is becoming recognised as an important one. Works investigating the effects of pedestrians on dynamic structures, like the Millennium Bridge and Daly's Bridge, are becoming more popular and such investigations forward the cause of standard implementation in industry.

\section{Methodology}

This project aimed to investigate the dynamic behaviour of Daly's Bridge and assess its structural health, as neither had been done before. Finite element modelling and instrumentation were two tools chosen to do this, which would complement each other and lead to a more holistic understanding of the structure.

For this project, as the first of its kind to be undertaken on this historic structure, we had to gather information from a variety of sources, old and new. When it became clear that no detailed drawings of the bridge were available, a survey to obtain all geometric data of the bridge had to be conducted which then allowed a 3D drawing to be created in the computer-aided design software AutoCAD. A visual inspection of each member of the structure for damage and corrosion was also carried out, and the members deemed structurally redundant, so damaged that they no longer held structural qualities, were systematically recorded. The material of the bridge was found to be steel, from an archived bill of quantities dating back to the time of construction. This document also helped in understanding the components used to connect different member of the bridge to one another, like the 8 sockets which anchored the cables to the ground.

\section{Finite Element Model}

In order to assess the vibration characteristics of the structure a finite element model was then constructed (Figure 4). This computer model was created using Strand 7 software, and it is a full geometric representation of the bridge, with each member assigned the appropriate material properties of steel and the connections between members were designed to represent the real, physical connections on the bridge. The damaged members, identified in the visual inspection, which included some members of the handrails and towers, were removed from the model for a more realistic representation. Changes were also made to the properties of steel used, in order to match the results of the model to the results from the testing of the as-built condition of the bridge, discussed below. 


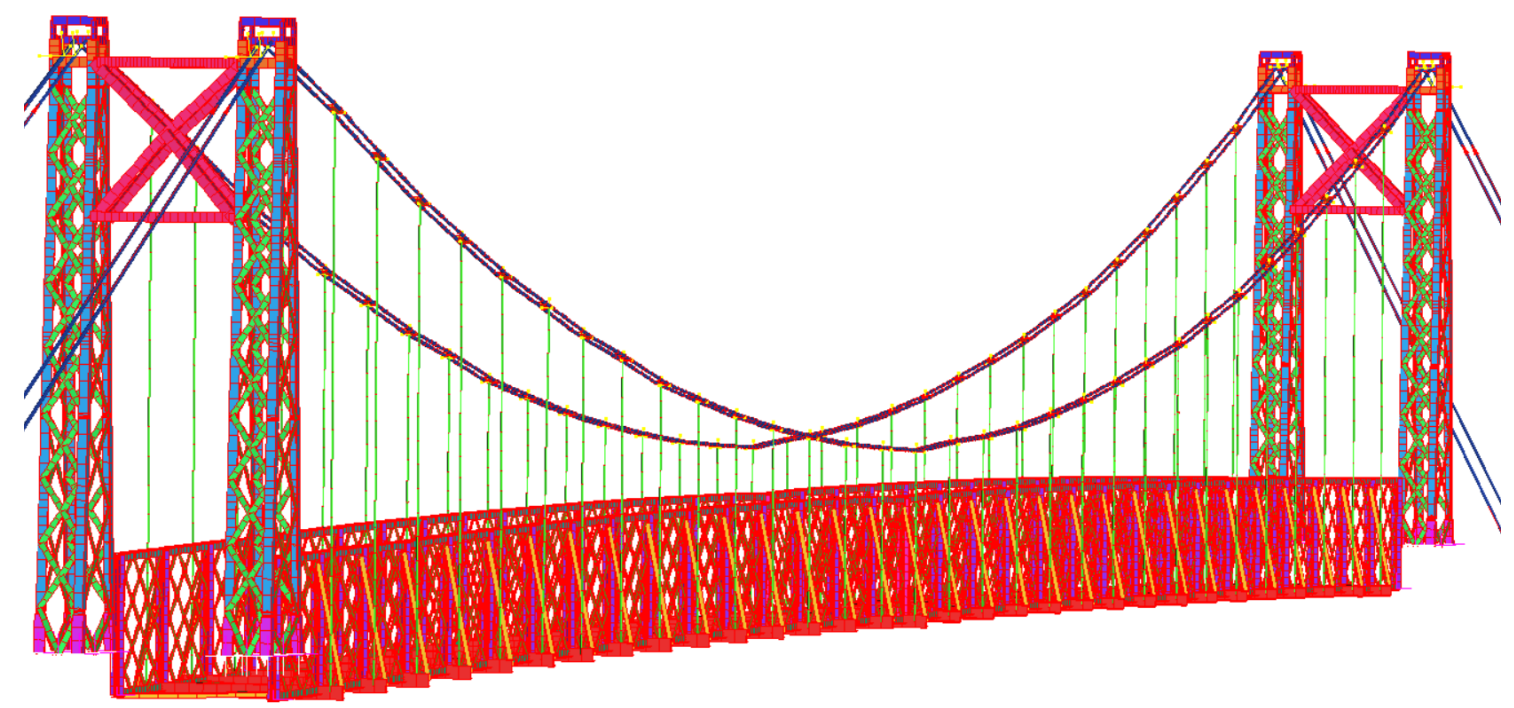

Figure 2: Detailed finite element model of Daly's Bridge. Source: Author

\section{Dynamic Testing}

With a model in place, a testing scheme was designed in order to get information about the bridge's behaviour for a range of excitations. Eight accelerometers were attached to the bridge at various positions along its span and on different structural components; one was placed on the cables at the centre span, another on the wooden walkway at the quarter span. These accelerometers recorded vertical accelerations of the structure over two hours. It was observed in its ambient position when no pedestrians were present, and for a range of controlled and uncontrolled pedestrian traffic, including individual walkers, groups of walkers and a lone cyclist. From this data, the velocity of the motion and the frequency of the motion could also be derived. This information was indicative of the structures behaviour and the reasons for that behaviour, and it also allowed comparisons to be made with the finite element model, which in turn had its accuracy improved to better represent the in situ condition of the bridge.

\section{Results}

The set of data from the days testing was analysed in order to assess, compare and rank, in terms of human comfort level, the response of the bridge to different excitation sources.

First, the acceleration is a key factor in what humans can perceive from a moving structure. The acceleration data for the periods of time where specific activity occurred on the bridge were extracted from the full data set, and these data sets were also analysed in terms of their frequency; this data is presented in Table 1. The acceleration recorded for a group of pedestrians traversing the bridge was the highest, and this matched observations made of the structure. Interestingly, a lone cyclist crossing the bridge barely excited the bridge 
Table 1: 1: Accelerations \& Frequencies of bridge under different excitations

\begin{tabular}{|l|c|c|}
\hline Excitation Source & $\begin{array}{l}\text { RMS Acceleration } \\
\left(\mathrm{m} / \mathrm{s}^{2}\right)\end{array}$ & $\begin{array}{l}\text { Peak Frequency } \\
(\mathrm{Hz})\end{array}$ \\
\hline Ambient Excitation (at rest) & .036 & 2.265 \\
\hline Cyclist & .048 & - \\
\hline Controlled Pedestrian 1 & .11 & 1.64 \\
\hline Controlled Pedestrian 2 & .38 & 2.25 \\
\hline Group of Pedestrians & .51 & $1.9-2.2$ \\
\hline
\end{tabular}

beyond the rest condition; due to the full contact between the bicycle and the walkway there was no impact loading, to which the bridge is notably sensitive.

In the comparisons of the natural frequency of the bridge, shown in Table 1 as the peak frequency under ambient excitation, and the forced frequency of pedestrians, the values are similar. The cases where the frequencies of excitation source and structure are closest, the highest amplitudes of acceleration were recorded.

In order to contextualize the data in terms of human perceptibility, we surveyed pedestrians as they crossed the bridge on the day of testing. They were asked to rank the motion of the bridge as one of the following; imperceptible, barely perceptible, distinctly perceptible, strongly perceptible or disagreeable. The majority ranked the bridge's motion as 'strongly perceptible', with people who often used the bridge noting that the response depends on the number and the pace of the users of the bridge at any given time, and the results of our testing corroborated this.

Finally, the matching of outputs of the finite element model to the actual responses of the bridge recorded during experimentation lead to a more realistic representation of the structure in its true condition. This matching is an important part of many structural health monitoring applications, as an accurate computer model can be used as a base line for assess future changes in the bridge, and assess the effect such damage might have on the structure, globally.

\section{Conclusion}

The matching of walker and bridge frequencies causes a resonance condition, which amplifies the motion of the bridge. Many 'Corkonians' and UCC students are familiar with the Shaky Bridge, and this investigation into its shakiness has offered insights into the reasons for its dynamic behaviour. The motion ranks as 'strongly perceptible' on the scale of human perception, and while a system of dampers could be implemented to reduce this motion, it would be expensive and ultimately unnecessary. With the locals' fondness for the Shaky Bridge as it currently stands, I'm sure Cork would have it no other way. 